\title{
Correction to: Endurance-oriented training program with children and adolescents on maintenance hemodialysis to enhance dialysis efficacy-DiaSport
}

\author{
Markus Feldkötter ${ }^{1} \cdot$ Sarah Thys $^{2} \cdot$ Anne Adams $^{3} \cdot$ Ingrid Becker $^{3} \cdot$ Rainer Büscher $^{4} \cdot$ Martin Pohl $^{5} \cdot$ Raphael Schild $^{6}$. \\ Lars Pape ${ }^{7}$. Claus Peter Schmitt ${ }^{8} \cdot$ Christina Taylan $^{9} \cdot$ Simone Wygoda $^{10} \cdot$ Günter Klaus $^{11} \cdot$ Henry Fehrenbach $^{12}$. \\ Carmen Montoya $^{13} \cdot$ Martin Konrad ${ }^{14} \cdot$ Heiko Billing ${ }^{15} \cdot$ Bettina Schaar $^{2} \cdot$ Bernd Hoppe $^{16,17}$ (I)
}

Published online: 9 September 2021

(C) IPNA 2021

\section{Correction to: Pediatr Nephrol.}

https://doi.org/10.1007/s00467-021-05114-8

The original version of this article unfortunately contained a mistake. During the process of typesetting, corrections to the figure legends of figs. 4 and 5 were not carried out completely. The publisher apologizes for this mistake. Below, the figures with the correct legends are presented below.

Publisher's note Springer Nature remains neutral with regard to jurisdictional claims in published maps and institutional affiliations.

The online version of the original article can be found at https://doi.org/ 10.1007/s00467-021-05114-8

\section{Markus Feldkötter}

Bernd Hoppe

bernd.hoppe@knz-bonn.de

1 Pediatric Nephrology Unit, University Children's Hospital Zurich, Zurich, Switzerland

2 Institute of Sport Science, Bundeswehr University Munich, Munich, Germany

3 Institute of Medical Statistics and Computational Biology, University Hospital of Cologne, Cologne, Germany

4 Pediatric Nephrology, Pediatrics II, University of Duisburg-Essen, Essen, Germany

5 Department of General Pediatrics, Adolescent Medicine and Neonatology, Medical Center - University of Freiburg, Faculty of Medicine, University of Freiburg, Freiburg, Germany

6 Pediatric Nephrology, University Children's Hospital, University Medical Center Hamburg-Eppendorf, Hamburg, Germany
7 Department of Pediatric Kidney, Liver and Metabolic Diseases, Hannover Medical School, Hannover, Germany

8 Division of Pediatric Nephrology, Center for Pediatric and Adolescent Medicine, Heidelberg, Germany

9 Pediatric Nephrology, Children's and Adolescents' Hospital, University of Cologne, Cologne, Germany

10 KfH Center of Pediatric Nephrology, St Georg Hospital, Leipzig, Germany

11 KfH Center of Pediatric Nephrology, Department of Pediatric Nephrology, Marburg, Germany

12 Pediatric Nephrology, Children's Hospital, Memmingen, Germany

13 KfH Center of Pediatric Nephrology, Children's Hospital Munich, Schwabing, Munich, Germany

14 Department of General Pediatrics, University Children's Hospital, Münster, Germany

15 University Children's Hospital, Tübingen, Germany

16 Children's Hospital, University of Bonn, Bonn, Germany

17 Kindernierenzentrum Bonn, Bonn, Germany 
A)

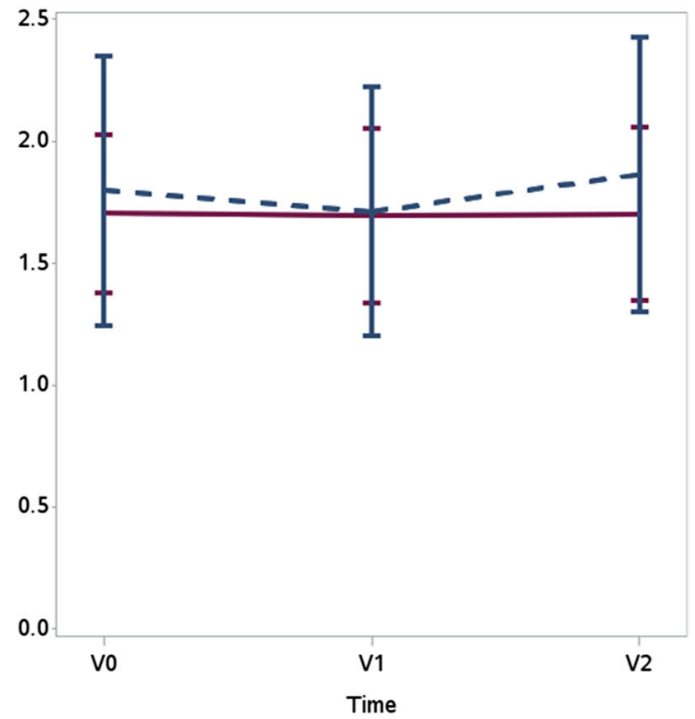

Fig. 4 Primary endpoint: A) Mean Single pool kt/V at different testing points, A) ITT = intention to treat population, B) LOCF = last observation carried forward. V0 Baseline, V1 post first intervention period
B)

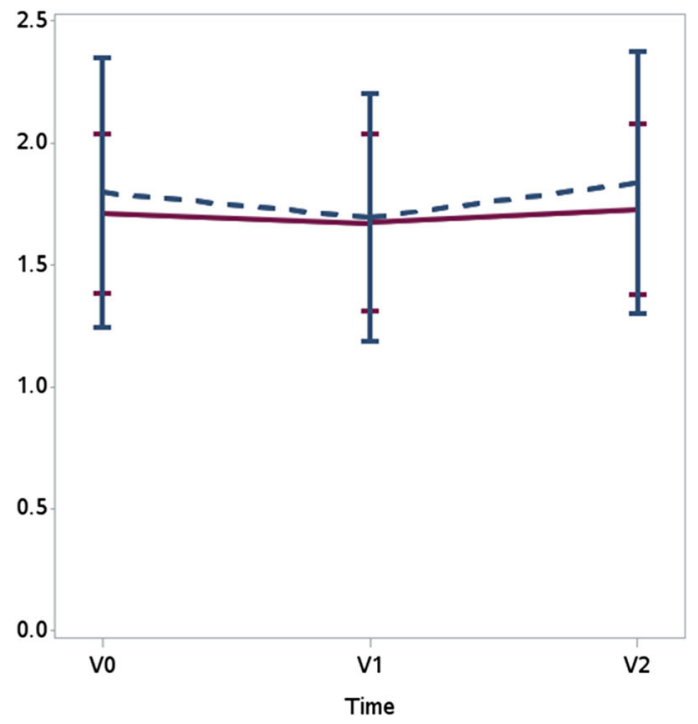

(12 weeks), V2 post second intervention period (24 weeks). Intervention (training) Waiting control - - - - - -
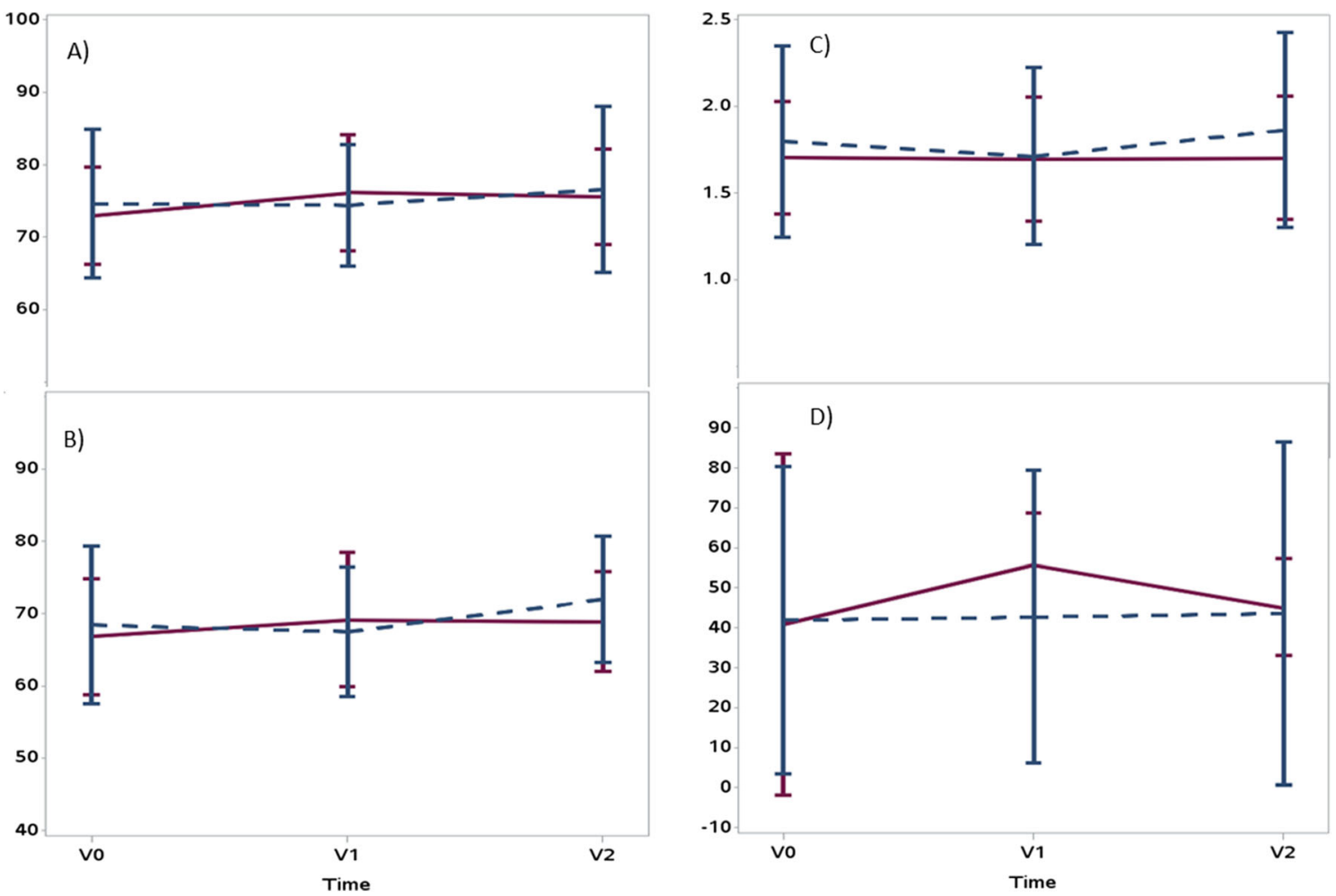

Fig. 5 Exemplary secondary endpoint: Mean elimination of low molecular substances - no changes between V0 to V2 for (A) blood urea, (B) serum creatinine, (C) serum phosphate and (D) mean double Pool Kt/V. Intervention (training)

Waiting control ------ 